\title{
Research on Effective Field Lines Detection and Tracking Algorithm in Soccer Videos
}

\author{
Shouzhong Zhang \\ Physical Education Department, Harbin Engineering University, \\ Harbin 150000, China \\ Zhangshouzhong03@163.com
}

\begin{abstract}
A novel field line detection method is proposed based on the directional information of field line. First, the special character of modern soccer playfield is considered, and a novel soccer field model is introduced which includes offside assistant lines. By designing a directional filter, these field lines can be accurately detected. Moreover, the field line detection and tracking for a clip is developed, which can further improve the performance of field line detection. In this paper, we integrate the basic theories of multimedia analysis, pattern recognition and computer vision into content analysis for sports video analysis. Through exploring several components such as object detection, camera calibration and tactic analysis, a systematical system is built up. The proposed algorithms can be not only used for soccer video analysis but also used for other sports video analysis. It provides a promising method for general multimedia analysis.
\end{abstract}

Keywords: Video content analysis, Soccer videos, Tactic analysis, Field lines detection

\section{Introduction}

The field is a main element in football match videos [1-2]. Almost all such match shots are on the background of the pitch. Furthermore, those highlights in the football videos occur in the field too. At some point, soccer games are activities that are constrained to a certain area. So football videos record images of activities in a defined area [3]. Players, judges and the rubber ball are all limited to such a place, even coaches and audiences are staying around it. Thus to analyze football videos, it's quite necessary to process and detect videos regarding field lines [4-5].

As mentioned above, we can classify shots to close-up, medium and long shots according to photographic intention and included objects. Of them, long shots are views against the pitch [6-7]. They often take up over ninety percent of all shots. For a specific purpose of reviewing what's contained in shots in a more detailed manner, we need to divide long shots into six different types like upper left, upper middle, upper right, lower left, lower middle and lower right part (Figure 1), to separately represent which pitch area a long shot is focusing on. However, that is not adequate for us to make profound investigations. Our final goal is to examine football match strategies. We need additionally to detect footballers' position and their movement trajectory, analyze their motional pattern as to eventually find out the strategy pattern which is decisive to the success of the ball competition. To reach it, we have to do in-depth analytics of long shots, in particular, to pick up lines of football venue of precisely and efficiently. The accurate extraction of those lines is premise to camera calibration and the fetch of targets' real track, which is the foundation to do strategy analysis. So in a word, field line extraction is basis to strategy analysis [8-9].

Field lines in the football playground are marked in white color [10]. Those lines refer to sidelines, ground lines, kick-off lines and penalty area lines, which are explicitly defined in the Laws of Football Game by Federation International de Football Association 
(FIFA). Because those lines are all white, so most detection algorithms use color information to detect field lines [11-12]. When cutting those white lines, we find it not easy to split them merely with color information as there are many complicated interference factors in the pitch. Those factors could be uneven colors of lawns in the field, grandstands in videos, blurred white lines, unstable team jersey color etc. [13] emphasized the geometric structure and features of images with mathematical morphology and geometry as the base. It developed a linear parameter method for automatic extraction of white lines in the field. To be specific, those white lines are apparently slender, long, and straight. Their color value is all higher than peripheral pixels in terms of RGB channels. They have good morphological properties. So it's suitable to use mathematical morphology for image intensification. Then, Top-Hat transform is applied to highlight white lines. Other morphologies are performed to eliminate grandstands and football players. In the end, a binary image of them is obtained.
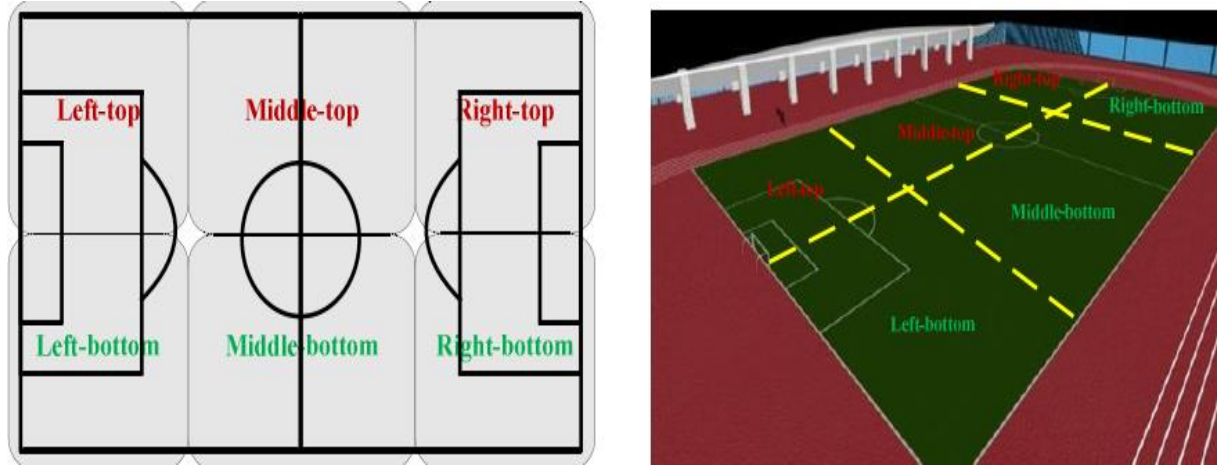

Figure 1. Six Different Scene Vision Lens

For the reason that most field lines are located surrounding the forbidden area, the forbidden area detection and that of field lines there are closely related topics. [14] employed three parallel lines as features to detect the penalty area in the field. In [15], with position relations between cathetus and curves in the field as feature, it proposed grammatical pattern recognition methods to describe those relations as to recognize seven parts in the field. The literature [16] utilized such information as field shape combined with SVM classifier to inspect highlights in the ball game. The author [17] detected position of the field by using features like rectilinear inclination and field shape. In [18], the information regarding straight lines was used to fix the region where the game is ongoing. [19] detected goal mouths in videos with the combined methods for both sideline detection and color region growing.

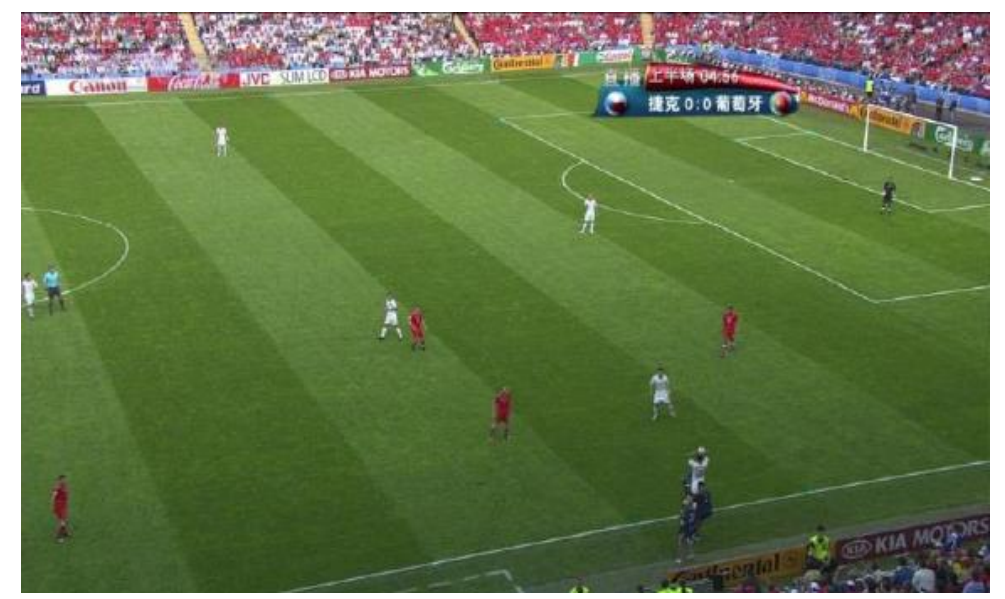

Figure 2. A Typical Long Shot in Soccer Video 
However, with the improvement of football playground, modern ball field contains other types of field lines, e.g. offside auxiliary lines for referees to judge offside. Those lines are not marked in white color, but formed with turfing techniques. Specifically, lawns are trimmed in two different directions to form two areas. Visually, one area is dark green and the other is light green. The two areas appear alternately, and that lines are formed in the intersection of different areas. Those lines can help referees decide whether there is offside. That's why they are called offside auxiliary lines. It is shown in Figure 2.

The auxiliary lines are greatly helpful to the accurate analysis of field areas. They can be used as calibrator for camera calibration and good to extract the true track of targets. The existing detection methods for field lines are based on color information. But for those field lines, their extraction requires other techniques instead of depending on the color information.

\section{Single Frame Detection of Field Lines Based on Direction Information}

The common methods for detecting field lines are performed with the color information that field lines are all white. But in our football field model, offside symbol lines among field symbol lines are not white. Being located in the intersection of deep green and light green areas, those offside lines are hardly detected by the direct use of color information. Offside lines have distinctive luminance variances. Their angles of inclination are limited to a small range like $35-55^{\circ}$ for the demi-court gauche and 125$145^{\circ}$ for the demi-court droit. Based on the priori knowledge, we develop a detection method based on directional filter banks. It is shown in Table 1

\section{Table 1. Field Lines Detection Algorithm Based on Directional Filter}

Input: The $i$ frame image $I_{\text {input }}^{i}$ in video sequence

Output: $\mathrm{N}$ field lines $L_{n}^{i}, n=1,2, \ldots, N$ in the image $I_{\text {input }}^{i}$

1 Separated the stadium area from image, image $I_{\text {input }}^{i}$ converted to grayscale images, And edge enhancement using Sobel operator, retaining only the stadium area, got the image $I_{\text {field }}^{i}$.

2 Enhanced the field line, used the filter matrix respectively four yuan directional filter banks $\left(H_{l}^{1}, H_{l}^{2}, H_{r}^{1}, H_{r}^{2}\right)$ respectively to filter for image $I_{\text {field }}^{i}$, filtered four images using conjunctive (or) operation to get filtered image $I_{\text {field }}^{i}$

3 Detected field lines, image $I_{\text {field }}^{i}$ was Hough transformed, detected the most significant $\mathrm{N}$ field lines $L_{n}^{i}, n=1,2, \ldots, N$

The proposed method has three steps: firstly splitting out the field area; as in football videos there are non-field areas like audience area, which will affect the detection precision of field symbol lines; also since the edge intensity of field area is much lower than the non-field ones. Sobel operator can be used for site edge enhancement, as shown in Figure 3(b); then images are cut into field area and audience area through preset threshold value (Figure 3(c)); secondly intensifying points in the field symbol lines; with priori knowledge about offside auxiliary line direction, we design two directional filters for respectively demi-court gauche and demi-court droit; see the equation (1) for filter matrix; points in offside auxiliary lines are intensified through differences, as seen in Figure 3(b); thirdly detecting field symbol lines; Hough transform is applied to detect $\mathrm{N}$ most remarkable field symbol lines, for which see Figure 3(d). 


$$
\begin{aligned}
H_{l}^{1} & =\left[\begin{array}{ccccccc}
1 & 2 & 3 & 4 & 0 & 0 & 0 \\
2 & 3 & 4 & 0 & 0 & 0 & 0 \\
3 & 4 & 0 & 0 & 0 & 0 & 0 \\
4 & 0 & 0 & 0 & 0 & 0 & 0 \\
0 & 0 & 0 & 0 & 0 & -4-3 \\
0 & 0 & 0 & 0-4-3-2 \\
0 & 0 & 0 & 4-3-2-1
\end{array}\right] ; H_{l}^{2}=\left[\begin{array}{cccccc}
1 & 2 & 3 & 4 & 0 & 0-4 \\
1 & 2 & 3 & 4 & 0-4-3 \\
2 & 3 & 4 & 0 & 0-4-3 \\
2 & 3 & 4 & 0-4-3-2 \\
3 & 4 & 0 & 0-4-3-2 \\
3 & 4 & 0 & 0-3-2-1 \\
4 & 0 & 0 & 4-3-2-1
\end{array}\right] ; \\
H_{r}^{1} & =\left(H_{l}^{1}\right)^{T} ; H_{r}^{2}=\left(H_{l}^{2}\right)^{T}
\end{aligned}
$$

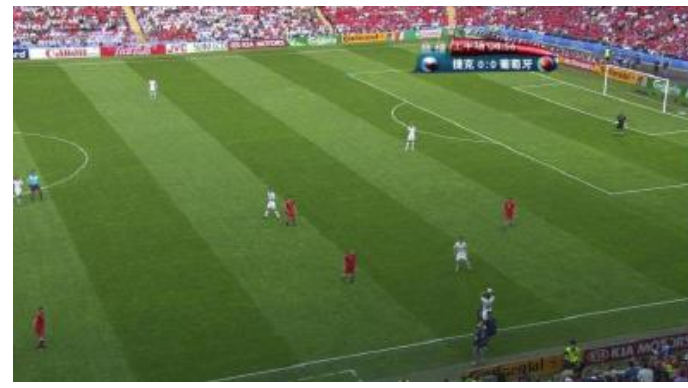

(a) The Input Image

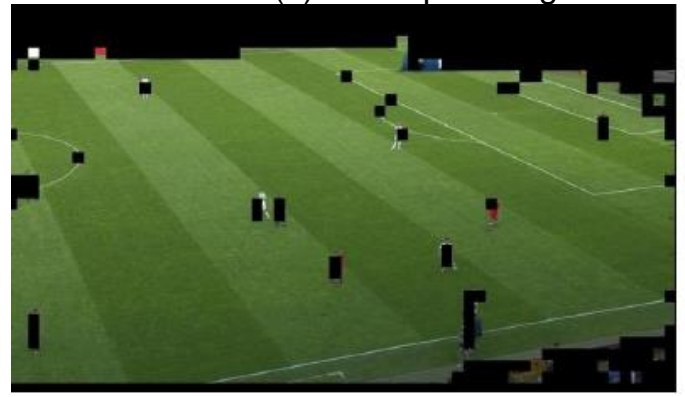

(c) Field Area of the Image

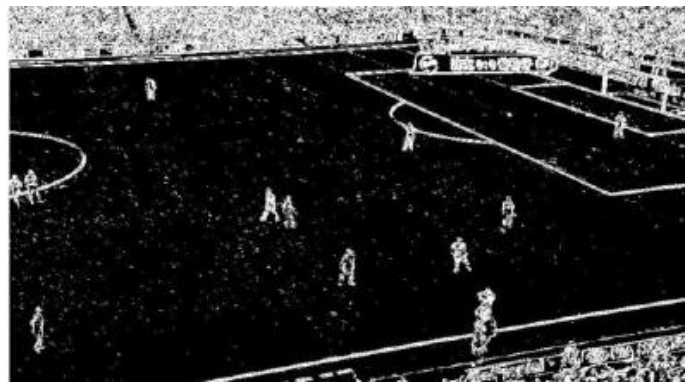

(b) Sobel Enhanced Images

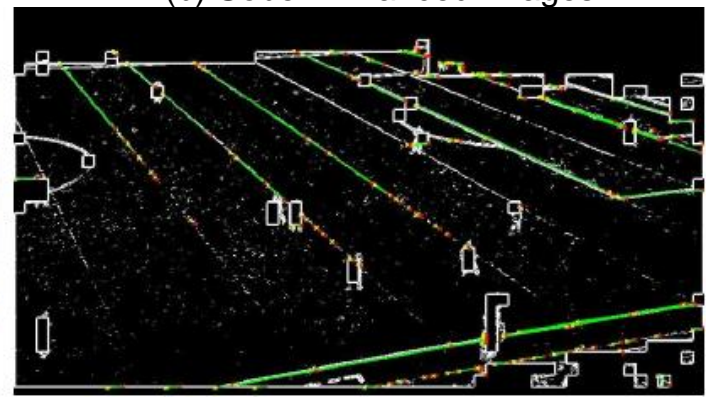

(d) Field Line Detection Image

Figure 3. Diagram of Field Lines Detection

\section{Track of Field Lines Based on Kalman Filtering}

\subsection{Independent Frame Field Lines Detection}

Many existing detection methods for field lines are executed by line color or direction information, as proposed in section 2. By Hough Transform, every $(\theta, \rho)$ in the space $P_{m}=\left(\theta_{m}, \rho_{m}\right)$ suggests a straight line $L_{m}^{f}$ in the image; a group of straight lines in the image correspond to a cluster of points in the space $(\theta, \rho)$; also every straight line has a Hough transform intensity, which indicates the possibility of the line existing in the image. In general, the clearer and longer the line is in the image, the bigger its intensity value is. So the real field lines in the image all have bigger intensity values, while those illusory noise field lines accordingly have smaller values. From the methods based on direction information, we can learn that after Hough transform is made for football match images, field lines appear in clusters. In one cluster, there is often one real field line and therefore others are virtual noise lines. In the methods based on direction information, K-means clustering algorithm is applied for clustering in the space $(\theta, \rho)$.After clustering, $\mathrm{N}$ 
classes are obtained, $C_{n}^{f}(n=1,2, \ldots, N)$. For each $C_{n}^{f}$, the straight line which has the biggest Hough transform intensity is selected for the real field line $L_{n}^{f}$.

As the image in the football broadcast videos is of low quality, the performance of the method is not satisfactory. As observed in Figure 4(a), there are many noise field lines in the image, which would be regarded mistakenly as field line if we perform directly clustering for them. To improve the accuracy of detection, here we present a preprocessing mechanism, allowing to remove lots of false field lines before clustering.

Generally speaking, real field lines in the image have two main directions of inclination, which are called two angles in the paper. They are corresponding to the sloping direction of side lines and end lines. The angle of real field lines must be parallel to one of the two main directions. Those not in the two directions are fictitious field lines. Hence, the priori knowledge can help us filter out effectively noise lines. The two angles in different frames in videos will change along with horizontal and vertical camera scanning. So it's necessity to adaptively determine the principal angles of different images. Here we take Gaussian mixture model to extract the angle of each image. To make it clear, we firstly get the Hough parameter $\left(\theta_{m}, \rho_{m}\right)(m=1,2, \ldots, M)$ of all field lines in one image; then use the Gaussian mixture model (GMM) with two components to create models for angle $\left(\theta_{m}\right)(m=1,2, \ldots, M)$. Considering directional angle in a circulation flow, we apply 2-dimensional GMM in the space $\left(\cos \left(\theta_{m}\right), \sin \left(\theta_{m}\right)\right)$ to make model for the angle. The mean values of two components in the GMM are considered to be two principal angles of the image. Field lines whose slanting angles are not consistent with the two principal ones are looked upon as noise lines and thus are removed.

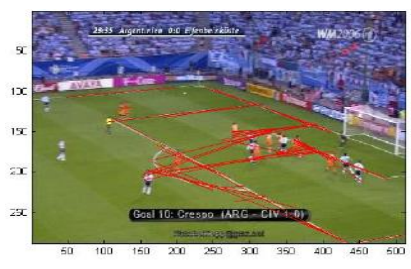

(a)

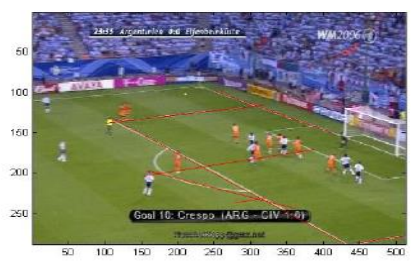

(b)

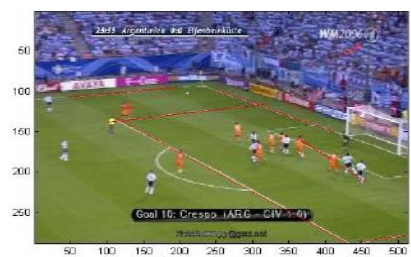

(c)

(a) Hough Transform is to Extract the Initial Field Lines

(b) K Mean Clustering Algorithm is to Extract the Initial Field Lines

(c) The Minimum Spanning Tree Clustering and Main Perspective of Gauss Mixture Model Filter Integrated Extraction Field Lines.

Figure 4. Extracted Real Field Lines

Used $\mathrm{K}$ mean value method in $(\theta, \rho)$ space for clustering on the field line based on directional information method. It is shown in figure 4 (b). This method by random initialization makes the final clustering result is not very stable. Therefore, this paper is to achieve cluster by the minimum spanning tree algorithm, this method can be hierarchical clustering of field line. Minimum spanning tree cluster algorithm is a method based on graph, each vertex in the graph represents a point $P_{m}$ in $(\theta, \rho)$ space, the weight between two points edges in map represent their Euclidean distance $D_{m n}=\left\|P_{m}, P_{n}\right\|$.After clustering, the line of the largest Hough transform strength in each class is used as a detection of the field lines, it is shown in figure 4 (c).

\subsection{The Field Lines Tracking Based on Video}


Football venues is relatively large, the camera cannot completely captures the entire field. In the football broadcast video, the camera is always adjusted to be able to capture the field regional wonderful events, so different frames of a video segment often contains the regional different venues. Therefore detected field lines from these frames are often inconsistent, for example, the currently visible field lines are disappeared at the back of the image. In order to compute the homography matrix, which must know the detected field line identification. The field line identification showed that it corresponds to football field model, determined the field lines identification problem called mark field line problems.

In [20], the model matching algorithm is used to solve this problem, because this method is proposed for the independent frame, for all frame tagging video clips in the field line will significantly reduce the efficiency of the system. As we know, video segments in the same field line in consecutive frames are often very close to the space location and continuity, the prior knowledge can help us to the efficient solution a video clip the line of field identification problem.

As mentioned before, an independent frame field line detection is not stable, some field lines was not detected because of occlusion, some detected field lines is noise line. Obviously the same field line in consecutive frames position continuity can also help improve the detection accuracy of field lines.

This paper presents an effective field line detection and tracking algorithm for tracking and identifying field line in a video clip, it is shown in Table 2 . This algorithm used video segments in the same field line position continuity to effectively suppress the false detection and missed detection field line.

\section{Table 2. The Detection Algorithm of Field Line Based on Directional Filter} Bank

Input: Image sequence of video fragment $I_{\text {input }}^{f},(f=1,2, \ldots, F)$

Output: Field lines sequence set $L_{\text {output }}^{n}, n=1,2, \ldots, N$

1 For image $I_{\text {input }}^{f}$, circulation $f=1,2, \ldots, F$;

(1) Extracted $O_{o b s}^{f}=\left\{L_{m}^{f}, m=1,2, \ldots, M\right\}$

Separated the stadium area from image, enhanced field line pixel; by Hough transform detected field line. Gauss mixture model extracted the main angle and filtering; with the minimum spanning tree method to obtain the final field line.

(2) Extracted $L_{\text {est }}^{f}=\left\{L_{n}^{f}, n=1,2, \ldots, M\right\}$

(a)For field line $L_{n}^{f-1}$ in $L_{e s t}^{f-1}$,by Calman filtering to Obtain $L_{n}^{f}$, circulation $n=1,2, \ldots, N$

(b) For $O_{o b s}^{f}$, The remainder of the observed field line $L_{m}^{f}$ to start tracking, circulation $m=1,2, \ldots, M$;

2 For the field line sequence $\left\{L_{n}^{f}, f=1,2, \ldots, F\right\}$, circulation $n=1,2, \ldots, N$

(a)Determined the start and end frame number each time period $\left\{\left(B_{n}^{t}, E_{n}^{t}\right),(t=1,2, \ldots, T)\right\}$.

(b) To obtain the output field line series $L_{\text {output }}^{n}=\left\{L_{n}^{f}, f=1,2, \ldots, F\right\}$.

\section{Experimental Analysis and Results}




\subsection{Single Frame Detection Result of Field Lines}

As mentioned previously, GMM-based filtering methods for principal angles and the clustering methods based on minimum spanning trees are both used to improve the performance of detecting field lines. Here we compare them with those based on K-means clustering. We choose randomly 300 images from six types of goals videos for the comparison. By the following equation, we calculate recall ratio $(\mathrm{R})$ and precision ratio $(\mathrm{P})$ as to evaluate qualitatively the effect of them.

$$
\begin{aligned}
& R=n_{c} /\left(n_{c}+n_{m}\right) \\
& P=n_{c} /\left(n_{c}+n_{f}\right)
\end{aligned}
$$

Where, $\mathrm{n} \mathrm{c}$ is the number of field lines detected in a right manner; $\mathrm{n} \mathrm{m}$ is the number of field lines not detected; $\mathrm{n} \mathrm{f}$ is the number of field lines detected falsely. In Table 3, the first column is result of methods based on K-means clustering; the second column is performance of clustering methods based on minimum spanning trees; the third column is result of the clustering method combing GMM-based principal angle filtering and minimum spanning trees. From Table 3 we learn that the clustering methods based on minimum spanning tree can improve recall ratio effectively; GMM-based principal angle filtering method is good for the improvement of precision ratio.

\begin{tabular}{|c|c|c|c|c|c|c|}
\hline \multirow[t]{2}{*}{$\begin{array}{l}\text { Video } \\
\text { clips }\end{array}$} & \multicolumn{2}{|c|}{$\begin{array}{l}\text { The algorithm based on } \\
\text { direction information }\end{array}$} & \multicolumn{2}{|c|}{$\begin{array}{l}\text { Minimum spanning tree based on } \\
\text { clustering method }\end{array}$} & \multicolumn{2}{|c|}{$\begin{array}{l}\text { The main filtering } \\
\text { method based on } \\
\text { GMM and } \\
\text { Minimum spanning } \\
\text { tree based on } \\
\text { clustering method }\end{array}$} \\
\hline & $\begin{array}{l}\text { precision } \\
\text { ratio }\end{array}$ & recall ratio & precision ratio & recall ratio & $\begin{array}{l}\text { precision } \\
\text { ratio }\end{array}$ & $\begin{array}{l}\text { recall } \\
\text { ratio }\end{array}$ \\
\hline S1 & $87 \%$ & $90 \%$ & $89 \%$ & $94 \%$ & $92 \%$ & $94 \%$ \\
\hline S2 & $83 \%$ & $80 \%$ & $85 \%$ & $94 \%$ & $90 \%$ & $95 \%$ \\
\hline S3 & $84 \%$ & $82 \%$ & $85 \%$ & $95 \%$ & $90 \%$ & $94 \%$ \\
\hline S4 & $87 \%$ & $88 \%$ & $90 \%$ & $96 \%$ & $92 \%$ & $95 \%$ \\
\hline S5 & $84 \%$ & $81 \%$ & $86 \%$ & $91 \%$ & $89 \% \%$ & $93 \%$ \\
\hline S6 & $81 \%$ & $79 \%$ & $87 \%$ & $90 \%$ & $92 \%$ & $93 \%$ \\
\hline Average & $84 \%$ & $83 \%$ & $87 \%$ & $93 \%$ & $90 \%$ & $94 \%$ \\
\hline
\end{tabular}

Table 3. Comparison of Single Frame Field Lines Detection Performance

\subsection{Detection Result of Field Lines in Video Clips}

The inspection of field lines in video clips was performed over video data regarding all goals moments. When the camera needs rapid adjustment, field lines would suddenly arise or disappear. So it becomes harder to perform the detection of such lines. To make it easier, from six types of attack pattern video clips, we selected as test data six clips for which the camera is adjusted heavily. Results are listed in Table 4. In the Table 4, SOLs means the summation of all field lines present in the image. Take for instance, if in the first frame image, there are 5 field lines and in the second frame image, there are 6field lines. SOLs is totally 11 for the two images.

As indicated in Table 4, the proposed method realized the precision and recall ratio at respectively $94 \%$ and $96 \%$. Where field lines were not detected correctly or tracked, the following can account for:

1. Some lines are on the edge of image, which could be too short to be detected;

2. When ambient brightness varies with time, some lines are of too low luminance to be inspected. 
Besides, we compared the performance of single frame method and video clip method for detecting field lines. In Table 4, it's obvious the later did better than the former by increasing the precision and recall ratio from $91 \% / 94 \%$ to $94 \% / 96 \%$.

Table 4. Comparison of Video Clips Field Lines Detection

\begin{tabular}{|l|l|l|l|l|l|}
\hline $\begin{array}{l}\text { Video } \\
\text { clips }\end{array}$ & \#SOLs & $\begin{array}{l}\text { Field line detection based on single } \\
\text { frame }\end{array}$ & $\begin{array}{l}\text { Field line detection based on } \\
\text { video clips }\end{array}$ \\
\cline { 3 - 6 } & & precision ratio & recall ratio & precision ratio & $\begin{array}{l}\text { recall } \\
\text { ratio }\end{array}$ \\
& & & & \\
\hline S1 & 450 & $92 \%$ & $94 \%$ & $96 \%$ & $95 \%$ \\
\hline S2 & 376 & $90 \%$ & $95 \%$ & $94 \%$ & $98 \%$ \\
\hline S3 & 392 & $90 \%$ & $94 \%$ & $95 \%$ & $96 \%$ \\
\hline S4 & 434 & $92 \%$ & $95 \%$ & $93 \%$ & $97 \%$ \\
\hline S5 & 438 & $89 \%$ & $93 \%$ & $94 \%$ & $96 \%$ \\
\hline S6 & 445 & $92 \%$ & $93 \%$ & $92 \%$ & $96 \%$ \\
\hline Average & 422 & $91 \%$ & $94 \%$ & $94 \%$ & $95 \%$ \\
\hline
\end{tabular}

\section{Conclusion}

Football field is an important object in the videos, for the effective detection of the target, extraction of its trajectory and analysis of related game strategy. The field analyzing is a basic but important step. The paper concerned about how to effectively and accurately pick up field lines. It developed a new field model, in which offside auxiliary lines are important element. Then a new detection method for field lines was designed based on the direction information. Further considering video continuity, we showed another method for detecting and tracking field lines in videos. It proved its effectiveness in decreasing false detection rate and loss rate through tracking mechanism. Unlike other methods, the proposed method took direction information as major features, which enables it to effectively find out offside auxiliary lines. Hence, it's particularly suitable for processing modern football match videos. The extraction of field lines provides conditions for camera calibration, target trajectory fetch as well as tactics analysis.

\section{References}

[1] White L. and Liu S., "A kind of automatic calibration method in broadcast soccer video camera based on pitch model," Acta automatica Sinica, vol. 3, (2012), pp. 321-330.

[2] Lin B., Liu Q., Wang Q. and Nie Y., "The soccer video shot classification method," Computer engineering and design, vol. 4, (2012), pp. 1467-1471.

[3] Yin W., Chen L. and Li W., "The site automatic detection method of sports video," The application of computer system, vol. 5, (2012), pp. 184-188.

[4] Zhang Y., Ding S., Wang J., Dai Y. and Chen M., "HMM fusion event detection based on multi modal," Journal of system simulation, vol. 8, (2012), pp. 1638-1642.

[5] Xu F., Zheng X. and Lv T., "The tracking method for soccer video in HOG and particle filter based on ball," Electronic Science and technology, vol. 9, (2013), pp. 36-40.

[6] With M., Ding L. and Maggie J. C., "Soccer video highlights the fusion of HCRF and AAM detection," Journal of computer research and development, vol. 1, (2014), pp. 225-236.

[7] Ding L. and Liu Y., "The soccer video highlights multidimensional semantic cues and HCRF model detection," Journal of computer aided design and computer graphics, vol. 11, (2013), pp. 1715-1724.

[8] Yu J., Zhang Q., Wang Z. and He Y., "Using the playback scene and emotion encouragement detection in soccer video highlights," Chinese Journal of computers, vol. 6, (2014), pp. 1268-1280.

[9] Yan G., Cui G. and Yu M., "Background modeling player Gauss mixed model based detection algorithm," Computer simulation, vol. 9, (2010), pp. 258-262.

[10] Duan X., Ji M., Wang F., Wei F. and Cao Q., "When 1. Rapid tennis ground wire detection based on Hough transform," The spread of science and technology, vol. 15, (2011), pp. 214-215.

[11] D. Farin, S. Krabbe and P. D. With, "Robust camera calibration for sport videos using court models," In SPIE Storage and Retrieval Methods and Applications for Multimedia, (2004), pp. 80-91. 
[12] D. Farin, J. Han and P. D. With, "Fast camera calibration for the analysis of sport sequences," In ICME, (2005), pp. 482-485.

[13] Liu G. and Dewey L. H., "The automatic extraction of a football field marking line," Journal of computer aided design and computer graphics, (2003), pp. 45-50

[14] A. Ekin, A. Tekalp and R. Mehrotra, "Automatic soccer video analysis and summarization," In TIP., vol. 12 no. 7, (2003), pp. 796-807.

[15] Y. Gong, T. Lim and H. Chua, “Automatic parsing of TV soccer programs," In ICMCS., (1995), pp. 167-174.

[16] Q. Ye, Q. Huang and W. Gao, "Exciting event detection in broadcast soccer video with mid-level description and incremental learning," In ACM MM. (2005), pp. 455-460.

[17] M. Bertini, A. D. Bimbo and W. Nunziati, "Model checking for detection of sport highlights," In Proc. ACM international Workshop on MIR. (2003).

[18] W. Jinjun, X. Changsheng and C. Engsiong, "Automatic replay generation for soccer video broadcasting," In Proc. ACM MM. (2004).

[19] X. Gao, Z. Niu and D. Tao, "Non-goal scene analysis for soccer video," In Neurocomputing. (2010).

[20] D. Lemire, “A better alternative to piecewise linear time series segmentation," In IAM SDM. (2007).

\section{Author}

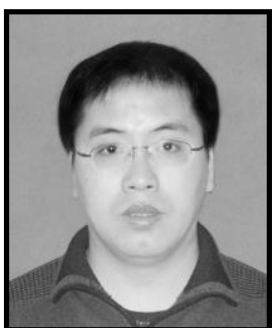

Shouzhong Zhang, He received his B.S degree in physical education from Jilin Institute of Physical Education. He got his M.S degree in physical education from Chengdu Sport University. He is a lecturer in Physical Education Department of Harbin Engineering University. His research interests include physical education and training. 
International Journal of Multimedia and Ubiquitous Engineering

Vol.10, No.7 (2015) 